\title{
Importance of physiology studies in producing flowers and ornamental plants
}

\author{
Ana Maria Mapeli ${ }^{1 *}$
}

The production of flowers and ornamental plants has stood out in national agribusiness, mainly due to market innovations and the increasing improvement in the quality and longevity of the products offered, becoming possible due to the knowledge of the physiological characteristics.

The vegetative and reproductive success of plant species mainly depends on carbon metabolism, since the increase in liquid photosynthesis and reduction in photorespiration and respiration promote a reduction in carbon consumption, in turn causing a favorable energy network which is necessary to accumulate chlorophyll, differentiate vegetative buds, floral evocation and flowering (Taiz et al., 2017); all factors which are considered in the market.

However, it is necessary to maintain a favorable water balance to perform these physiological processes, which depends on absorption and transpiration in order to avoid flowers and leaves wilting, as well as the production of ethylene. In addition, proper development depends on mineral nutrition, since most symptoms of nutritional deficiency detract from the appearance and quality of vegetables and increase susceptibility to attack by phytopathogens.

Considering that senescence is the last stage of plant development, it is relevant to delay this event; its primary sign is yellowing of the leaves caused by the increase in ethylene production which promotes chlorophyll degradation, synthesis and/or revelation of carotenoids, reducing the quality of the entire plant's appearance.

It is worth mentioning that the accumulation of photoassimilates is reflected in the fresh and dry mass of the plant which directly interferes in longevity, mainly of species sold as cut flowers, as it represents a reserve which in turn will be used during breathing to produce the energy used in floral opening or in forming metabolic intermediates.

Growth and differentiation also depend on plant regulators belonging to different hormonal classes, including auxin, gibberellin, cytokinin, ethylene and abscisic acid, among others, which interfere with cell division and elongation, directly influencing vigor and height, which in turn is reflected in vase harmony, an essential characteristic for potted plants.

These substances also contribute to acclimatization processes which enable plant establishment in adverse environmental conditions (Kollist et al., 2019). This fact makes it possible to decentralize floricultural production to regions further away from the traditional producing poles.

The vegetable response to the environment depends on both non-enzymatic (carotenoids, vitamins, phenolic compounds) and enzymatic defense systems, with an emphasis on enzymes such as catalase, superoxide dismutase, peroxidase, and glutathione reductase. These physiological characteristics enable many species to be cultivated under high irradiance and high temperatures, enabling open-air cultivation which simplifies the production process and significantly reduces the initial investment by the producer.

Plant defense can also occur through secondary metabolites which have protective functions against unfavorable biotic and abiotic factors, as well as contributing to plant reproduction. Phenolic compounds stand out in this context, more specifically flavonoids, which are responsible for the petal color, a fundamental characteristic in the consumer's choice when purchasing ornamental flowers.

These changes in the physiological and agronomic characteristics of various cultures can positively modify their metabolism, being an alternative for the emergence of new varieties. This adds value in ornamental flower and plant production, since it enables presenting a product which will attract the customer's attention. This scenario is important because the ornamental market is extremely dynamic and constantly demands new innovations/ products.

Based on physiological knowledge, it is possible to carry out a genetic improvement program which is compatible with the demands of the consumer market, aiming at more efficient cultivation, reducing losses and increasing productivity, which in turn guarantees economic advantages and biodiversity preservation, in addition to obtaining plants which have commercial value and are competitive with existing hybrids. The new tools available offer new opportunities for manipulating the genetic and functional bases involved in the production under conditions of biotic and abiotic stresses, since the platforms available on the market involving the "omics" enable extensive characterization of the transcriptome, metabolome and the proteome.

The beauty of a plant as a whole stands out among the main changes, represented by good coloration of the flowers and leaves, size and number of flowers and good leaf formation, as well as the cultural and commercial

${ }^{1}$ Federal University of Western Bahia, Center for Biological and Health Sciences, Barreiras-BA. *mmapeli@ufob.edu.br ${ }^{0000-0002-6028-1989}$ 
advantages including ease of cultivation, development time, resistance to pests and diseases, adaptation to different environmental conditions such as drought, flood or saline soils, morphological changes, increase in the flower size, the flower durability and expansion of transport and storage time (Boutigny et al., 2020).
Despite this innovation, flower breeding programs generally prioritize ornamental traits over agronomic traits, which depends on physiological knowledge. The association of these two parameters could reduce the production costs of these new cultivars, the incidence of pests and diseases, and favor plant growth and differentiation.

\section{Reference}

TAIZ, L.; ZEIGER, E.; MÜLLER, I.M.; MURPHY, A. Fisiologia e Desenvolvimento Vegetal, 6ed. Porto Alegre: Artmed, 2017.

KOLLIST, H.; ZANDALINAS, S.I.; SENGUPTA, S.; NUHKAT, M.; KANGASJÄRVI, J.; MITTLER, R. Rapid responses to abiotic stress: Priming the landscape for the signal transduction network. Trends in Plant Science, v.24, p.25-37, 2019. doi.org/10.1016/j.tplants.2018.10.003

DE, L.C. Improvement of ornamental plants -a review. International Journal of Horticulture, v.7, n.22, p.180-204, 2017. doi: 10.5376/ijh.2017.07.0022.

BOUTIGNY, A.; DOHIN, N.; PORNIN, D.; ROLLAND, M. Overview and detectability of the genetic modifications in ornamental plants. Horticulture Research, v.7, n.11, p.1-12, 2020. doi.org/10.1038/s41438-019-0232-5 